\title{
SODIUM AESCINATE INJECTION FOR SKIN FLAP TRANSPLANTATION OF HAND OR FOOT IN CHILDREN
}

\author{
Wei Liyou $^{1}$, Liu Zhikui ${ }^{2}$, Zhang Hongwei ${ }^{*}$, Wang Guoqiang ${ }^{1}$ \\ ${ }^{1}$ Department of Orthopaedics, The Second Hospital of Tangshan, Tangshan City, Hebei Province, China \\ 063000; ${ }^{2}$ Department of Orthopaedics, Kailuan general hospital, Tangshan City, Hebei Province, China \\ 063000; ${ }^{3}$ Department of Intensive Care Unit, The second Hospital of Tangshan, Tangshan City, Hebei \\ Province, China 063000
}

*Corresponding Author's E-mail: zhanghw200087@163.com

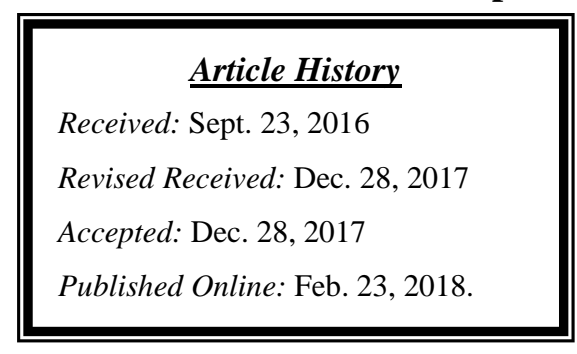

\section{Abstract}

Background: The purpose of this study is to evaluate the efficiency and safety of sodium aescinate injection for treating children suffering transplanted flap in children hand or foot.

Materials and Methods: Ninety children patients with transplanted cutaneous nerve nutrition vascular flaps in hand or foot were selected and divided into "treatment" and "control" groups randomly by computer. The treatment group was prescribed intravenous sodium aescine injection and conventional therapy. The control group was only offered conventional therapy. Seven days following treatment, cumulative wound drainage, swelling of flap and adverse reactions were recorded. One month subsequent to treatment, two-point discrimination of flap was recorded. At the outset of treatment (0-d) and 1, 3, 7-days following treatment, the concentrations of C-reactive protein (CRP) and malondialdehyde(MDA) in venous blood were tested.

Results: At the outset of treatment, there was no statistically significant difference between the two groups in terms of CRP and MDA ( $P>0.05)$. At 3 and 7 day intervals following treatment, the concentrations of CRP and MDA in treatment group were lower than those in control group $(\mathrm{P}<0.05)$. The treatment Group's wound drainage, swelling of flap and two-point discrimination of flap was better than those recorded in control group $(\mathrm{P}<0.05)$.

Conclusion: Sodium aescine injection can improve the concentrations of CRP and MDA in venous blood, reduce wound drainage, and promote flap sensory recovery. This has an effective and safe application for treating children with swelling in their hands or feet.

Keywords: Sodium Aescine Injection, Child, Hand or Foot, flap, Swelling.

Abbreviations: CRP, C-reactive protein; MDA, malondialdehyde; ATP, adenosine triphosphate.

\section{Introduction}

Children's tissue injuries of their hands or feet are common during emergency. The selected flap with a similar tissue structure of hand or foot is used to repair the defect. This not only reconstructs the function, but also restores its 
normal appearance, which is beneficial to the early recovery and long-term adaptability of children (Wei et al., 2013; Wei et al., 2014c; Wei et al., 2015). Flap is the most common and effective method in repairing orthopedic deformities and tissue defect. Yet, the ischemia-reperfusion injury often occurs after flap transplant. This can cause partial or total flap necrosis, and seriously affects the surgical outcome (Harder et al., 2008). Therefore, during the postoperative period, the effective medicine for preventing the occurrence of complications, and promoting flap survival should be given priority and be taken seriously.

Sodium aescinate is extracted from Borneo sub-dried seeds, which is a natural plant. It has a significant effect on anti-inflammatory, anti-exudative and reducing swelling (Matsuda et al., 1997). Sodium aescine restores normal capillary permeability, increases venous tension, and improves microcirculation. Sodium aescine also has a strong affinity for scavenging free radicals. It has a phenolic hydroxy in its molecular structure, which explains why it attracts scavenging oxygen free radicals. Sodium aescine was originally prescribed for treating cerebral edema with satisfactory results (Mingrino et al., 1978; Rivano et al., 1969). Presently, it has been widely applied for the treating a wide assortment of swelling caused by trauma, burns and surgery(Zhang et al., 2012), and likewise venous reflux disorders (Siebert et al., 2002; Carrasco et al., 2007; Raffetto et al., 2011).

There are scant reports regarding sodium aescine in treating children's diseases. The purpose of this study is to verify the efficacy of sodium aescine for improving swelling, draining and sensory ability in children following flap transplants and evaluate the safety of this drug in treating children patients.

\section{Materials and Methods}

\section{General information}

From January 2010 to December 2013, 90 children with tissue defects in hand or foot were selected. Tissue defects were repaired using cutaneous nerve nutrition vascular flaps, and surgical procedures were approved by senior professional doctors. Sample Group consisted of 49 males and 41 females ranging in age from 5 to 14 years old. Sixty-two children had hand injuries while twenty-eight children had foot injuries. Fifteen children were treated using flap surgery electively and seventy-five children treated using flap during emergency. These children patients were assigned numbers according to the time they admitted. Again these groups were sub-divided into treatment and control groups by computer-generated random selection. Their distribution ratio was 1: 1, 45 children in each group. There was no statistically significant difference regarding cutaneous nerve nutrition vascular flap nor statistically significant differences in terms of gender, age, time of injury, surgery, trauma site, damage severity, location (left or right) etc.. Lastly there was no statistically significant difference in terms of surgeon's work experience or professional title.

\section{Exclusion criteria}

All children had not had any dehydration medicines in recent one week or any glucocorticoid medicines in the most recent month. Excluded children were those with heart disease, previous heart surgeries, kidney damage, kidney failure, renal insufficiency, hepatic insufficiency, acquired immunodeficiency virus (HIV) infections, abnormal clotting condition, water electrolyte imbalance and insufficient blood circulation.

\section{Ethical considerations}

All children and their parents signed a consent form for participating in this study. This research was approved by the Medical Reproductive Ethics Committee of authors' Hospital. 


\section{Intervention}

The children with tissue defects in their hands or feet were prescribed conventional therapy. Following flap transplantation, homeostasis and analgesia, anti-infection, nutritional support, maintaining water and electrolyte balance treatment were measured. The treatment group was given an additional sodium aescine injection (Shandong Luye Pharmaceutical Co., Ltd., Shandong, China) one day following transplantation (within 24 hr), dosage of sodium aescine injected according to body weight being $0.2 \mathrm{mg} / \mathrm{kg}$, which dissolved in $250 \mathrm{ml} 0.9 \%$ sodium chloride injection. Maximum daily dose did not exceed 20mg. This continued once per day, 7 days for a full course of treatment.

\section{Variable assessed}

At the outset of treatment (within 24 hours) and at 1, 3, 7-days following treatment, C-reactive protein (CRP) and malondialdehyde (MDA) in venous blood were tested. Seven days after treatment, cumulative wound drainage, the swelling of flap and adverse reactions were recorded. One month after treatment, two-point discrimination of flap was recorded during the follow-up. CRP was tested by automatic biochemical analyzer. MDA was determined by thiobarbituric method. Cumulative wound drainage (7 days) included direct wound drainage volume and the amount of fluid intake gauze, the amount of fluid intake gauze bases on area or volume of infiltration.

\section{Two-point discrimination test}

All child patients had the purpose and methodology explained to them before testing obtaining and getting their cooperation. During testing, the examiner fixed the patient's limbs and instructed them to close their eyes. While the two needle detectors were descending, the patient was required to indicate when one or two points were felt- quickly. As the distance between these two detectors decreased, patient was required to state when the two points could not be properly distinguished. Replying correctly more than seven of ten times test was accepted as the correct resolution, the minimum distance at this test is the two-point discrimination detection value (Zhang et al., 2005).

\section{Swelling criteria}

With reference to swelling (Liao et al., 2009), 0 degree of swelling is when the skin of flap has no swelling, striae of skin exists and elasticity of skin is normal. I degree of swelling is when there is mild swelling of flap compared to normal skin, but there are still sticks and swelling center height $\leq 0.5 \mathrm{~cm}$ compared with contralateral normal limb. II degree of swelling is when skin tension increases, striae of skin disappears, but no blisters and swelling center height is in 0.5 to $1 \mathrm{~cm}$ compared with contralateral normal limb. III degree of swelling is when skin of flap has significant swelling, tension blisters, swelling center height $\geq 1 \mathrm{~cm}$ compared with contralateral normal limb.

\section{Safety evaluation}

All patients were involved with safety. Adverse effects were recorded and included dizziness, palpitation, nausea, diarrhea and skin allergies. Other indicators were tested before and after treatment- primarily liver function, kidney function, electrolytes, urine, stool routine and others.

\section{Statistical analysis}

Statistical software SPSS17.0 (SPSS software Inc., USA) was used to analyze data. Measurement data was presented as (mean \pm standard deviation). T test was used for two groups. The $\mathrm{x}^{2}$ test was used to analyze numeration data. The Mann-Whitney test was used for non- parametric. $\mathrm{P}<0.05$ was regarded as denoting statistically significant difference. 


\section{Results}

\section{Comparison of basic characteristics}

All child patients completed this study. There was no statistically significant difference $(\mathrm{P}>0.05)$ between the two groups in terms of sex (Male/Female), age, trauma site (Hand/Foot) and location(Left/Right). Seven days after treatment, the drainage volume of wound and swelling of flap in the treatment group was better than those in the control group and statistical differences were significant $(\mathrm{P}<0.05)$. One month after treatment, two-point discrimination of flap in treatment group was better than that in the control group, this difference being statistically significant $(\mathrm{P}<0.05)$ as provided in Table 1.

Table 1: The basic characteristics in two groups

\begin{tabular}{|c|c|c|c|c|c|c|c|}
\hline \multirow{2}{*}{ Group } & $\begin{array}{c}\text { Sex } \\
\text { (cases) }\end{array}$ & \multirow{2}{*}{$\begin{array}{l}\text { Age }\left(\overline{\boldsymbol{x}}_{ \pm \mathrm{s}}\right. \\
\text { years old })\end{array}$} & $\begin{array}{l}\text { Location } \\
\text { (cases) }\end{array}$ & Site (cases) & \multirow{2}{*}{$\begin{array}{c}\text { Drainage } \\
\text { volume }(\mathrm{ml})\end{array}$} & \multirow{2}{*}{$\begin{array}{c}\text { Two-point } \\
\text { discrimination } \\
(\mathrm{mm})\end{array}$} & \multirow{2}{*}{$\begin{array}{l}\begin{array}{l}\text { Swelling } \\
\text { (cases) }\end{array} \\
\text { 0/ I / II / III }\end{array}$} \\
\hline & $\begin{array}{l}\text { Male / } \\
\text { Female }\end{array}$ & & Left / Right & Hand / Foot & & & \\
\hline Control & $26 / 19$ & $9.10 \pm 2.80$ & $20 / 25$ & $30 / 15$ & $72.25 \pm 22.70$ & $9.73 \pm 3.68$ & $2 / 20 / 22 / 1$ \\
\hline Treatment & $23 / 22$ & $9.30 \pm 2.71$ & $17 / 28$ & $32 / 13$ & $58.11 \pm 20.51$ & $7.46 \pm 3.74$ & $7 / 25 / 13 / 0$ \\
\hline$x^{2} / t / Z$ & $x^{2}=0.403$ & $\mathrm{t}=0.344$ & $x^{2}=0.413$ & $x^{2}=0.207$ & $\mathrm{t}=2.881$ & $t=2.902$ & $Z=2.471$ \\
\hline p-value & $\mathrm{p}=0.525$ & $\mathrm{p}=0.732$ & $\mathrm{p}=0.520$ & $\mathrm{p}=0.649$ & $\mathrm{p}=0.005$ & $\mathrm{p}=0.004$ & $\mathrm{p}=0.013$ \\
\hline
\end{tabular}

\section{Comparison of CRP}

At the outset of treatment (0-d), there was no statistically significant difference between the two groups in term of CRP $\left(\mathrm{t}_{0}=0.997, \mathrm{P}>0.05\right)$. One day following treatment, the concentrations of CRP in both groups rose to a peak. Likewise the CRP declined between 3 to 7-days following treatment, Concentrations of CRP in the treatment group were again lower than those in the control group $\left(t_{1}=3.272, t_{3}=8.597, t_{7}=8.003, P<0.05\right)$. As shown in Figure 1 .

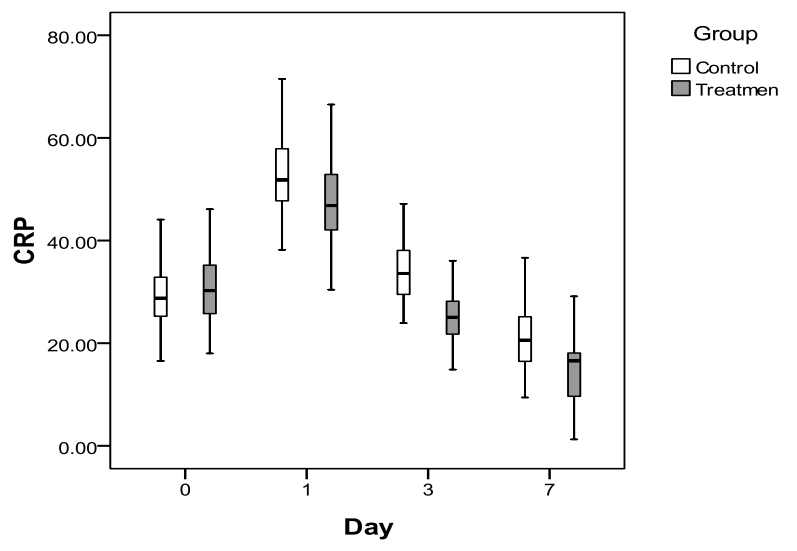

Figure 1: Comparison of CRP in two groups

Note: All children patients with tissue defects in their hands or feet were prescribed conventional therapy after flap transplantation. This included hemostasis and analgesia, anti-infection, nutritional support, maintaining water and electrolyte balance treatment. The treatment group was given an additional sodium aescine injection for 7 days $(0.2 \mathrm{mg}$ $/ \mathrm{kg}$, it dissolved in $250 \mathrm{ml} 0.9 \%$ sodium chloride injection). The maximum daily dosage did not exceed $20 \mathrm{mg}$, once a day. The unit of CRP is $\mathrm{mg} / \mathrm{L}$

\section{Comparison of MDA}

At the beginning of treatment $(0-\mathrm{d})$, there was no statistically significant difference between the two groups in 
term of MDA ( $\left.\mathrm{t}_{0}=0.112, \mathrm{P}>0.05\right)$. One day following treatment, the concentrations of MDA in two groups rose to their peak and later the MDA declined between 3 to 7-days following treatment. The concentrations of MDA in treatment group were lower than those in control group $\left(t_{1}=9.569, t_{3}=10.046, t_{7}=7.420, P<0.05\right)$. As shown in Figure 2.

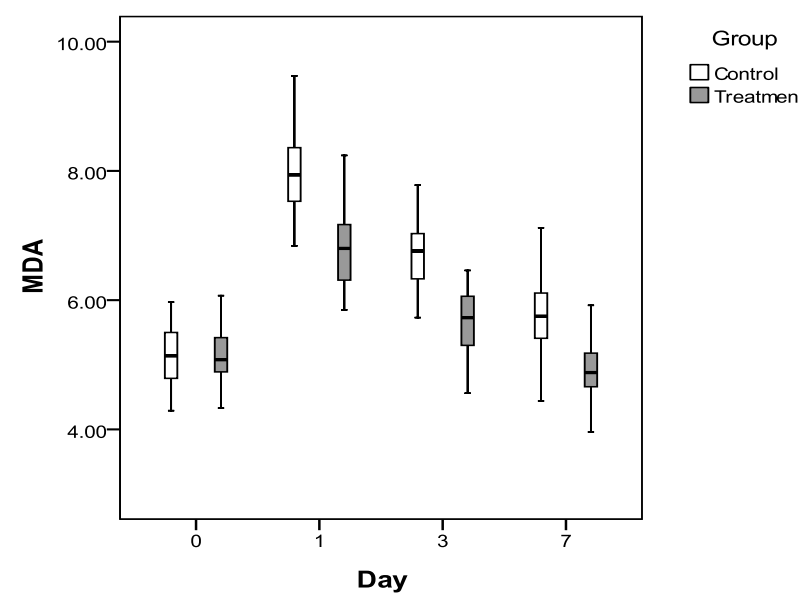

Figure 2: Comparisons of MDA in two groups.

Note: All subjects with tissue defects in their hands or feet were prescribed conventional therapy after flap transplantation. This consisted of homeostasis and analgesia, anti-infection, nutritional support, maintaining water and electrolyte balances. The treatment group was provided an additional sodium aescine injection for $7 \mathrm{days}(0.2 \mathrm{mg} / \mathrm{kg}$, it dissolved in $250 \mathrm{ml} 0.9 \%$ sodium chloride injection). The maximum daily dosage did not exceed $20 \mathrm{mg}$. The unit of MDA is $\mathrm{nmol} / \mathrm{mL}$.

\section{Safety Evaluation}

There were no adverse reactions such as dizziness, palpitation, nausea, diarrhea and skin allergies in either group. There was no liver malfunctioning, kidney failure or electrolyte imbalance and abnormal urine and stool routine. One child patient in the treatment group had local irritation pain while the intravenous medicine was dripping. However, after slowing the injection rate, these symptoms disappeared. Intravenous injection rate should be 15 to $30 \mathrm{drops} / \mathrm{min}$, and the initial infusion rate should be slightly slower. There was no statistically significant difference between these two groups in terms of the safety $(\mathrm{P}>0.05)$.

\section{Discussion}

Soft tissue defects in children's hands or feet were oftentimes accompanied by damage to the tendon and/or bones. If these injuries are not handled properly, it will affect the development and function of the hands and feet. Children are at the growing and maturing stage and consequently preserving limb length and restoring function is the basic prerequisite for treatment (Wei et al., 2014a; Wei et al., 2014b). Since children have small hands and feet, these tissues have a limited ability for transplanting and this likewise requires consummate repair technology. Choosing the appropriate flap is conducive to body function recovery and is helpful for restoring function and purpose. During flap grafting, the stimulated wounds produce an inflammatory response and accordingly such mediums as bradykinin, histamine and interleukin are released resulting in the edema of flap and the exudation of wound. Following surgery, an ischemic reperfusion type injury of flap can infuse an increased rate release of inflammatory mediators and oxygen free radicals. This then directly aggravates the tissue damage. An open wound accompanied with severe swelling of flap can lead directly to flap occurred necrosis. Therefore, treatment priorities following flap transplantation is the timely elimination of inflammatory mediators and free radicals, lifting the edema of flap and improving micro-circulation.

Sodium aescinate is the sodium salt of triterpenoid saponins with multi ester bond. It has the effects of anti-inflammatory, improving swelling and scavenging oxygen free radicals. This has been widely applied in the 107 
clinical treatment of various forms of etiologies edema (Fu et al., 2005; Wang et al., 2011). The metabolism of Sodium aescinate with its effects of anti-inflammatory and improving edematous was similar to adreno cortico hormones (Zhang et al., 2012; Wang et al., 2013). Sodium aescinate increases capillary permeability, improves local micro-circulation, increases vein circulation while effectively eliminating local tissue edema. It also has a strong role of scavenging oxygen free radicals, thereby reducing its harmful effects on tissue while thus realizing neuroprotection. Other related studies have concluded that sodium aescinate reduces denosine triphosphate (ATP) in cellular under hypoxic conditions, and maintains the function of cell permeability, thereby alleviating the impact of injury on tissues and cells (Kang et al., 2013; Janssens et al., 2000).

CRP is acute phase proteins compounded by liver cells when the body is subjected to microbial invasion or when tissue is stimulated by inflammatory mediators. Under normal circumstances, it is a trace protein in either serum or plasma. CRP is a very sensitive indicator in assessing tissue damage and infection (Sierra et al., 2004). Research indicates when concentrations of CRP are positive related to cell damage and concentrations of CRP were greater in more serious injuries (Li et al., 2008). During flap transplantations, involving both operational and traumatic stress, systemic inflammatory responses in children were accordingly aggravated. CRP was marker of trauma and inflammation so consequently the concentration of CRP increased following surgery. The major active compound in extracts from horse chestnut (Aesculus hippocastanum), has shown clinically significant anti-inflammatory activity (Sirtori, 2001; Huang et al., 2015; Wang et al., 2009). The concentrations of CRP in treatment group were lower than those in control group.

Oxygen free radicals have a strong oxidation resistance. This acts on the various components of the cell, leading to lipid peroxidation. The end product of lipid peroxidation is MDA. Therefore, the concentration of a body's MDA reflects the extent of lipid peroxidation. Under normal physiological conditions, there is only a small amount of oxygen free radicals in the body. Since antioxidant enzymes and antioxidants can remove excess oxygen free radicalsIschemia-reperfusion injuries usually occurs after flap transplantation. An increased amount of oxygen free radicals are produced causing serious damage to the body's normal cells and tissues. Some research purports that sodium aescinate has protective effects on ischemic reperfusion in adults, and can reduce levels of free radicals (Yang et al., 2011). At 1, 3 , and 7-days following treatment, concentrations of MDA in the treatment group were lower than the control group (P $<0.05)$. In this study, these results again indicated sodium aescinate's role in scavenging oxygen free radicals in children, the outcomes were consistent with other clinical and animal experiment (Wang et al., 2012; Wei et al., 2011).

In this study, drainage of wound and swelling of flap in treatment group were better than those in control group and these differences were statistically significant $(\mathrm{P}<0.05)$. Sodium aescinate's ability as being anti-leakage and suppressing swelling. A Two-point discrimination test for a treatment group having better results than the control group caln be interpreted as sodium aescinate removing excess oxygen free radicals. This reduces the damage caused by inflammation factors and oxygen free radicals and therein promoted neurological recovery of skin flap and the swelling of flap resulting in faster recovery.

Generally, sodium aescinate is prudently dispensed to children. Recently however, it has been generally recommended for treating children related diseases. The safety of treatment group was parallel to that of the control group and sodium aescinate was dispensed cautiously to children with security. When this medicine is prescribed to children, the rate of injection must be controlled and the dosage should be adjusted according to the child's weight and likewise avoiding the medicine being injected into the subcutaneous.

\section{Conclusion}

Sodium aescinate can improve the concentrations of both CRP and MDA in children's venous blood, reducing wound drainage, and promoting early sensory recovery of the flap. It has an effective and safe application for treating children with swelling in their hands or feet.

Conflicts of interest: The authors declare that there are no conflicts of interest related to the publication of this paper. 108 
Author Contributions: Wei LY and Zhang HW designed the subject. Wei LY drafted and revised the article. Wang GQ and Liu ZK collected cases and performed the statistical analysis. All authors approved the final version of the article.

\section{References}

1. Carrasco, O.F.; and Vidrio, H. (2007). Endothelium protectant and contractile effects of the antivaricose principle escin in rat aorta. Vascular Pharmacology. 47(1): 68-73.

2. Fu, F.; Hou, Y.; Jiang, W.; Wang, R.; and Liu, K. (2005). Escin: inhibiting infammation and promoting gastrointestinal transit to attenuate formation of postoper-ative adhesions. World Journal of Surgery. 29(12): 1614-1620.

3. Harder, Y.; Amon, M.; Laschke, M.W.; Schramm, R.; Rücker, M.; Wettstein, R.; Bastiaanse, J.; Frick, A.; Machens, H.G.; Küntscher, M.; Germann, G.; Vollmar, B.; Erni, D and Menger, M.D. (2008). An old dream revitalised:preconditioning strategies to protect surgical flaps from critical ischaemia and ischaemia-reperfusion injury. Journal of Plastic, Reconstructive \& Aesthetic Surgery. 61(5): 503-511.

4. Huang, G.L.; Shen, D.Y.; Cai, C.F.; Zhang, Q.Y.; Ren, H.Y and Chen, Q.X. (2015). $\beta$-escin reverses multidrug resistance through inhibition of the GSK3 $\beta / \beta$-catenin pathway in cholangiocarcinoma. World Journal of Gastroenterology. 21(4): 1148-1157.

5. Janssens, D.; Delaive, E.; Houbion, A.; Eliaers, F.; Remacle, J.; and Michiels, C. (2000). Effect of venotropic drugs on the respiratory activity of isolated mitochondria and in endothelial cells. British Journal of Pharmacology. 130(7): 1513-1524.

6. Kang, J.; Gong, P.; Ren, Y.B.; Gao, D.N.; and Ding, Q.L. (2013). Effect of $\beta$-sodium aescinate on hypoxia-inducible factor- $1 \alpha$ expression in rat brain neurons after cardiopulmonary resuscitation. Chinese Journal of Emergency Medicine. 22(4): 373-378. [Chinese article]

7. Liao, Q.D.; Liu, X.; Wu, Z.; Wang, S.J.; Huang, J.J.; Zhong, D.; Weng, X.J. and Yan, A. (2009). Effect of extract of horse chestnut seeds (Aescuven forte) on limb trauma. Central South Pharmacy. 7(3): 224-226. [Chinese article]

8. Li, W.X.; Tao, S.Y. and Li, G.P. (2008). Variation and significance of C-reactive protein in trauma patients. Practical Clinical Medicine. 9(3): 1-6. [Chinese article]

9. Matsuda, H.; Li, Y.; Murakami, T.; Ninomiya, K.; Yamahara, J and Yoshikawa, M. (1997). Effects of escins Ia, $\mathrm{Ib}$, IIa, and IIb from horse chestnut, the seeds of Aesculus hippocastanum L., on acute inflammation in animals. Biological \& Pharmaceutical Bulletin. 20(10): 1092-1095.

10. Mingrino, S. and Scanarini, M. (1978). Anti-edema effect of escin at the cerebral level. Minerva Anestesiologica. 44(6): 355-360.

11. Raffetto, J.D. and Khalil, R.A. (2011). Ca2+-Dependent Contraction by the Saponoside Escin in Rat Vena Cava. Implications in Venotonic Treatment of Varicose Veins. Journal of Vascular Surgery. 54(2): 489-496.

12. Rivano, C. and Rosadini, G. (1969). Escin in the treatment of cerebral edema. Clinical and electroencephalographic study with correlographic analysis and spectrum of action. Minerva Neurochirurgica. 13(1): 92-94.

13. Siebert, U.; Brach, M.; Sroczynski, G. and Berla, K. (2002). Efficacy, routine effectiveness, and safety of horsechestnut seed extract in the treatment of chronic venous insufficiency. A meta-analysis of randomized controlled trials and large observational studies. International Angiology. 21(4): 305-315.

14. Sierra, R.; Rello, J.; Bailén, M.A.; Benítez, E.; Gordillo, A.; León, C. and Pedraza, S. (2004). C-reactive protein used as an early indicator of infection in patients with systemic inflammatory response syndrome. Intensive Care Medicine. 30(11): 2038-2045.

15. Sirtori, C.R. (2001). Aescin: pharmacology, pharmacokinetics and therapeutic profile. Pharmacological Research. 44(3): 183-193. 
16. Wang, H.; Zhang, L.; Jiang, N.; Wang, Z.; Chong, Y and Fu, F. (2013). Anti-inflammatory effects of escin are correlated with the glucocorticoid receptor/NF- $\mathrm{B}$ signaling pathway, but not the COX/PGF2 $\alpha$ signaling pathway. Experimental and Therapeutic Medicine. 6(2): 419-422.

17. Wang, T.; Fu, F.; Zhang, L.; Han, B; Zhu, M. and Zhang, X. (2009). Effects of escin on acute inflammation and the immune system in mice. Pharmacological Reports Pr. 61(4): 697-704.

18. Wang, T.; Jiang, N.; Han, B.; Liu, W.; Liu, T.; Fu, F. and Zhao, D. (2011). Escin attenuates cerebral edema induced by acute omethoate poisoning. Toxicology Mechanisms and Methods. 21(5): 400-405.

19. Wang, Y.K.; Han, J.; Xiong, W.J.; Yuan, Q.Y.; Gu, Y.P.; Li, J.; Zhu, Z.; Zhang, H.; Wang, C.J. (2012). Evaluation of in vivo antioxidant and immunity enhancing activities of sodium aescinate injection liquid. Molecules. 17(9): 10267-10275.

20. Wei, L.Y.; Wang, G.Q.; Chen, H. and Zhang, H.W. (2014a). Clinical application of first dorsal metacarpal artery flap for repair of thumb tissue defect in emergency. Chinese Journal of Bone and Joint Injury. 29(3): 257-259. [Chinese article]

21. Wei, L.Y.; Wang, G.Q.; Chen, H. and Zhang, H.W. (2015). Effects of the sural nerve and saphenous nerve nutrient vascular island flaps in repairing pediatric ankle or foot soft tissue defect. Chinese Journal of Medical Aesthetics and Cosmetology. 21(2): 23-25. [Chinese article]

22. Wei, L.Y.; Wang, G.Q.; Yi, F.; Zhang L. and Zhang, H.W. (2013). Radial dorsal neurocutaneous vascular flap repair pediatric thumb tissue defect. Chinese Journal of Hand Surgery. 29(6): 383-384. [Chinese article]

23. Wei, L.Y.; Wang, G.Q.; Zhou, H.Y.; Chen, H. and Zhang, H.W. (2014b). Three reconstruction feeling the pedicled island flap repaired children thumb injury in emergency. Chinese Journal of Plastic Surgery. 30(3): 257-259. [Chinese article]

24. Wei, L.Y.; Zhang, H.W.; Zhao, G.; Wang, G.Q.; Sun, C.J. and Chen, H. (2014c). Saphenous neurocutaneous vascular flap and external fixator in treatment of tibial fracture with soft tissue defect in children. Orthopedic Journal of China. 22(24): 2277-2280. [Chinese article]

25. Wei, T.; Tong, W.; Wen-ping, S.; Xiao-hui, D.; Qiang, X.; Tian-shui, L.; Zhi-fang, C.; Hong-fang, J.; Li, N.; Bin, Z.; Jun-bao, D. and Bao-ming, G. (2011). The impact of sodium aescinate on acute lung injury induced by oleic acid in rats. Experimental Lung Research. 37(10): 585-599.

26. Yang, G. and Cui, S.Y. and Huang, L.B. (2011). Protective effects of the preconditioning with different doses of sodium aescinate on tourniquet-induced ischemic reperfusion. Chinese Journal of Integrative Medicine. 17(9): 542-545.

27. Zhang, L.; Wang, H.; Wang, T.; Jiang, N.; Yu, P.; Liu, F.; Chong, Y and Fu, F. (2012) Potent anti-inflammatory agent escin does not affect the healing of tibia fracture and abdominal wound in an animal model. Experimental and Therapeutic Medicine. 3(4): 735-739.

28. Zhang, W.B.; Hong, G.X.; Wang, F.B. and Kang, H. (2005). Effect of sensory reeducation in combination with methycobal on sensory restoration of peripheral nerve injury. Chinese Journal of Physical Medicine and Rehabilitation. 27(10): 617-620. [Chinese article]. 